\title{
LETTERS
}

\section{Bedside Rounding Strategies Used by Bedside Teachers}

\author{
Darlene LeFrancois, MD and Sharon Leung, MD
}

Albert Einstein College of Medicine, Bronx, NY, USA

$\mathrm{J}$ Gen Intern Med 28(9):1130

DOI: $10.1007 / \mathrm{s} 11606-013-2461-\mathrm{x}$

(c) Society of General Internal Medicine 2013

To the Editors:-We applaud Dr. Gonzalo and colleagues' efforts to compile reported elements that respected bedside teachers use in preparing and conducting rounds. ${ }^{1}$ However, we have concerns that the rounds described are not fully engaging patient-centered bedside rounds practices.

In comparison to the definition of beside rounds previously published by Gonzalo, ${ }^{2}$ we were surprised to find that the definition of bedside rounds in the current study did not necessarily require the history presentation in the presence of the patient. We pondered the reasons for the shift and find this current definition unfavorable toward achieving patient-centered care. Without engaging the patient to verify and elaborate history, attending physicians are fully reliant on trainee reports. A thoughtful, focused, and compassionate presentation at the bedside which engages the patient is inherent to an accurate patientcentered approach.

Attending physicians are described preparing for rounds; "nearly all attending physicians reviewed written notes, EMR, and if possible, evaluated patients directly...'I look up information... from the previous day. I decide what I am going to focus on..." Clinicians cannot model patientcentered progressive problem solving if they are anchored to a diagnosis and/or management plan before they have seen the patient. We think the ability to engage with the patient and learners authentically in a collaborative thought process regarding the accurate diagnoses and best patientcentered management is now handicapped by the fact that the attendings have already done the thinking without these other parties.
Finally, although it is not completely clear how educational value was defined for patient prioritization and selection, it is inferred that patients who did not have "challenging diagnoses" were considered low educational value and would likely have bedside rounds deferred. The number of patients in the hospital with multiple chronic conditions is staggering. ${ }^{3}$ Exacerbations of these diagnoses may not be "unfamiliar," and clinical decision making may not be "challenging" since treatment protocols exist. However, the process of shared decision-making of disease management is vital to these patients.

It is clear that patients prefer a less authoritarian and more of a shared decision-making approach to their medical care. ${ }^{4}$ In fact, patient-centered and value-based care is now written as part of the Affordable Care Act. This cannot be achieved without affirming patients' values through patientclinician communication. How can we transform inpatient care to achieve this? While we need additional strategies, bedside rounds, when well defined, prepared, and applied, can move us in the right direction.

Corresponding Author: Darlene LeFrancois, MD; Albert Einstein College of Medicine, 111 East 210th Street, NW3 Blue Zone, Suite 359, Bronx, NY 10467, USA (e-mail: dlefranc@montefiore.org).

\section{REFERENCES}

1. Gonzalo JD, Heist BS, Duffy BL, et al. The art of bedside rounds: a multi-center qualitative study of strategies used by experienced bedside teachers. J Gen Intern Med. 2013;28(3):412-20.

2. Gonzalo JD, Chuang $\mathbf{C H}$, Huang G, Smith C. The return of bedside rounds: an educational intervention. J Gen Intern Med. 2010;25(8):7928.

3. Vogeli C, Shields AE, Lee TA, et al. Multiple chronic conditions: prevalence, health consequences and implications for quality, care management, and costs. J Gen Intern Med. 2007;22(Suppl 3):391-5.

4. Frosch DL, May SG, Rendle KAS, et al. Authoritarian physicians and patients' fear of being labeled 'difficult' among key obstacles to shared decision making. Heal Aff. 2012;31(5):1030-8. 